\title{
Tangence
}

\section{La réalité de la fausse lettre : observations pour une épistémologie appliquée de l'épistolarité}

\section{Manon Brunet}

Numéro 45, octobre 1994

Authenticité et littérature personnelle

URI : https://id.erudit.org/iderudit/025823ar

DOI : https://doi.org/10.7202/025823ar

Aller au sommaire du numéro

Éditeur(s)

Tangence

ISSN

0226-9554 (imprimé)

1710-0305 (numérique)

Découvrir la revue

Citer cet article

Brunet, M. (1994). La réalité de la fausse lettre : observations pour une épistémologie appliquée de l'épistolarité. Tangence, (45), 26-49.

https://doi.org/10.7202/025823ar d'utilisation que vous pouvez consulter en ligne.

https://apropos.erudit.org/fr/usagers/politique-dutilisation/ 


\title{
La réalité de la fausse lettre: observations pour une épistémologie appliquée de l'épistolarité
}

\section{Manon Brunet}

\author{
Mais cela est inventé pour exprimer le \\ vrai mieux encore que ne le fait la \\ réalité. \\ (Lettre de Gabrielle Roy à sa sœur \\ Bernadette, 20 janvier $1963^{1}$ )
}

La lettre donne l'illusion d'être plus vraie que toute autre forme d'écriture, y compris même les autres écritures du "moi", telles que désignées par le philosophe Georges Gusdorf ${ }^{2}$ : journal intime, autobiographie, mémoires. C'est du moins l'impression que Lucienne a, lorsqu'elle se résout à livrer au grand public les lettres d'amour idyllique qu'Alain Grandbois lui écrivait en 19321933: "Parler de soi est souvent d'une insurmontable difficulté, on ne se voit jamais comme nous voient les autres. [...] tout ce qui touche notre nature, notre caractère, devrait être abordé avec beaucoup de modestie. Une modestie que l'on ne trouve pas souvent dans les autobiographies ou les journaux intimes [...]. On y décèle un besoin de s'embellir, une tendance à cacher la vérité " ${ }^{3}$. Cette illusion tiendrait justement du fait que le "moi", dans la lettre au destinataire, ne peut indéfiniment mentir à l'autre qui le connaît mieux que lui-même.

Mais qui est ce "moi" qui, dans la lettre plus que dans le roman par exemple, se donnerait aussi entièrement et purement à lire, même dans la plus belle complicité amoureuse, amicale, filiale? Dans le cadre d'une phénoménologie de la quotidienneté

1 Gabrielle Roy, Ma chère petite scur: lettres à Bernadette, 1943-1970, édition préparée par François Ricard, Montréal, Boréal, 1988, p. 70-71.

2 Georges Gusdorf, Lignes de vie 1: les écritures du moi, Paris, Éditions Odile Jacob, 1991, $430 \mathrm{p}$.

3 Lucienne, "Introduction ", dans Alain Grandbois, Lettres à Lucienne, Montréal, l'Hexagone, 1987, p. 12. 
et d'une analyse du matériau autobiographique, Herman Parret ne craint pas de poser une question fort dérangeante pour le mythe de l'intimité épistolaire, en intitulant toute une section de son livre sur Le sublime du quotidien: "Ma vie": est-ce bien à moi qu'est la vie?" ${ }^{4}$ Le statut de vérité donné assez spontanément à la lettre ne dépend-il pas de la profonde croyance que nous avons que celui qui l'écrit est plus réel que le personnage fictif dont la vie est mise en scène dans le roman ou au thêâtre? que l'épistolier parle plus de lui-même dans la lettre que le romancier ne peut le faire à travers ses personnages? C'est pourquoi, les lettres de Madame de Sévigné, quoiqu'on dise sur leur degré d'intention esthétique, paraîtront toujours plus vraies que celles, inventées, de la religieuse portugaise ou des Liaisons dangereuses; que les lettres de Laure Conan adressées à son protecteur Henri Raymond Casgrain ne souffriront jamais la comparaison ontologique et morale sur leur authenticité avec celles d'Angéline de Montbrun. La lettre aurait le pouvoir, plus ou moins circonstanciel, de se passer de la médiation, filtre obligé de la fiction.

L'autre aspect de la lettre, le plus convaincant quant à la vérité exprimée dans la lettre, est que la lettre raconte des histoires plus vraies que celles du roman. Toutes les petites histoires racontées dans les lettres, où le quotidien est bien nommé et situé, servent même, de manière privilégiée, à raconter l'histoire d'une vie personnelle, celle du signataire de la lettre. Ainsi se construisent la mémoire individuelle et sociale, l'autobiographie et la biographie. Cependant, les éléments discursifs de la lettre ne sont pas tous considérés comme aussi vrais les uns que les autres: anecdotes, dates, noms et interprétations des événements relatés ne reçoivent pas tous le même degré de vérité.

Dans un premier temps, je ferai une très courte analyse de la réflexion qui a été développée sur la réalité et l'écriture de la lettre, depuis le fameux débat des années 1970 mené par Bernard Bray et Roger Duchêne, autour des lettres de Madame de Sévigné. Ce débat n'a pas eu d'équivalent au Québec. Le bilan, depuis celui fait par Bernard Beugnot en $1974^{5}$, ne semble pas avoir été poursuivi, bien que dans plusieurs ouvrages et articles

4 Herman Parret, Le sublime du quotidien, Paris, Éditions Hadès-Benjamins, 1988 , p. 39.

5 Bernard Beugnot, "Débats autour du genre épistolaire : réalité et écriture", Revue d'bistoire littéraire de la France, $\mathrm{n}^{\circ} 2$ 2, 1974, p. 195-202. 
disséminés se trouve toujours énoncé de manière explicite ou implicite ce paradoxe épistolaire perpétuel sur la vérité et l'esthétique de la lettre. Je terminerai par des propositions en vue d'une lecture sociologique de l'épistolaire qui tienne davantage compte du lecteur de la lettre. Il me semble que dans les études littéraires $^{6}$, cette perspective a été beaucoup moins développée, étant donné le statut réservé à la lettre considérée comme une cuvre très personnelle et en général sans grande valeur esthétique. Ce statut que les études littéraires lui ont réservé a fait justement que les approches psychanalytiques, formalistes, thématiques et, maintenant pragmatiques, ont eu plus de faveur jusqu'à maintenant. Ce qui nous amènerait à examiner autrement cette délicate question de l'authenticité des correspondances, lesquelles font l'objet, à l'heure actuelle, de plusieurs analyses et éditions.

Contre des réflexes parfois trop bien établis en matière de lecture, d'usage et d'interprétation de la lettre - ce document qui semble si immédiatement compréhensible par tout un chacun -, je suggérerais ensuite une réflexion méthodologique ouverte sur la connaissance a priori que nous avons de la lettre et qui nous la fait prendre pour plus vraie que d'autres formes d'écriture esthétique, ce que j'appellerais le sens commun de l'épistolarité. Je me référerai au corpus épistolaire littéraire québécois. Pour mieux comprendre ce qu'on attend d'une vraie lettre, j'examinerai les réflexes habituels du lecteur vis-à-vis de ce qu'il perçoit comme une fausse lettre. Ce qui donnera lieu à une certaine typologie de la fausse lettre, établie selon l'interprétation fournie soit par les correspondants eux-mêmes (auteurs/lecteurs), soit par les autres appelés lecteurs extérieurs.

6 Ailleurs, des sociologues s'intéressent de plus en plus, pour l'étude des rapports sociaux et de leurs représentations, à des matériaux aussi "personnalisés" que la lettre, reprenant ainsi la voie tracée par un des penseurs les plus influents de la sociologie moderne, Émile Durkheim qui avait pris pour objet sociologique une pratique aussi apparemment individualiste que Le suicide (1897). On lira, dans ce sens, avec intérêt et profit les travaux de Roch Hurtubise sur l'interprétation sociologique d'un corpus de lettres amoureuses, dont "Les amoureux et l'intime: à propos du discours et du silence amoureux", dans Manon Brunet, Serge Gagnon (dir.), Discours et pratiques de l'intime, Québec, Institut québécois de recherche sur la culture, 1993, p. 149-163. 


\section{Le débat sur la fausse lettre: sincérité, esthétique et illusion textuelle}

Une typologie succincte des fausses lettres nous apprend qu'elle est tributaire de deux perceptions différentes de l'acte épistolaire. Le débat sur le statut de l'authenticité de la lettre a jusqu'ici porté essentiellement sur le statut réservé à l'auteur de la lettre. Ainsi, les concepts d'intention artistique, de conscience littéraire ont abondamment été utilisés pour suggérer des éléments de réponse. On se souviendra des distinctions apportées en 1971 par Roger Duchêne dans le débat sur l'intentionnalité des lettres de Madame de Sévigné. L'auteur épistolaire est "celui qui se soucie plus d'un public éventuel que de celui à qui il est censé écrire" ${ }^{7}$ et ce qui, chez lui, est mis à contribution, "c'est l'ingéniosité $[\ldots]$ non la sincérité, la vérité" ${ }^{8}$, tandis que l'épistolier est "celui qui ne tient pas compte de l'existence du public" $"$ et qui a le souci de la vérité, tel Madame de Sévigné ${ }^{10}$. De telle sorte que "ce soupçon de littérarité porté sur la "sincérité" des sentiments qui s'y expriment "11, tel qu'identifié encore récemment par Vincent Kaufmann, entraînerait la réduction de toute pratique esthétique, y compris celle du jeu de la séduction, à une pratique du faux plutôt que du faux-semblant.

Roger Duchêne répondait ainsi à Bernard Bray qui, quant à lui, proposait alors pour la première fois une analyse du système épistolier, entendu comme l'observation dans la correspondance même de la marquise de la "place importante [qui] est toujours réservée à la question des motivations et des conditions de l'écriture " ${ }^{12}$. Son approche formaliste et structuraliste innovait, comme

7 Roger Duchêne, "Réalité vécue et réussite littéraire: le statut particulier de la lettre", Revue d'bistoire littéraire de la France, vol. LXXI, $\mathrm{n}^{\circ} 2$, mars-avril 1971, p. 177.

8 Ibid., p. 181.

9 Ibid., p. 177.

10 Cette thèse avait été défendue par Roger Duchêne un an plus tôt dans son ouvrage intitulé Réalité vécue et art épistolaire, 1: Madame de Sévigné et la lettre diamour, Paris, Bordas, 1970, 417 p.: "Nous avons cru que le meilleur était d'accepter comme véridiques, au moins à titre d'hypothèse de travail, les confidences de l'épistolière" (p. 12).

11 Vincent Kaufmann, L'équivoque épistolaire, Paris, Éditions de Minuit, 1990, p. 157.

12 Bernard Bray, "Quelques aspects du système épistolaire de $\mathrm{M}^{\text {me }}$ de Sévigné", Revue d'bistoire littéraire de la France, $\mathrm{n}^{\text {os }} 3-4,1969$, p. 494. 
l'a bien vu Bernard Beugnot ${ }^{13}$, puisqu'elle ne laissait plus place à l'interprétation du seul historien dans la détermination de la finalité de l'acte épistolaire. Elle suggérait effectivement de se référer plutôt à la désignation de cette finalité par l'épistolier lui-même se faisant, se montrant dans son propre système textuel épistolier. Mais cette position, comme celle de Duchêne, nous renvoie toujours du côté de l'auteur de la lettre.

Je voudrais tenter de répondre à cette même question, sur la façon de reconnaître la vérité épistolaire, en renversant le point de vue, c'est-à-dire en situant le lieu d'observation privilégiế plutôt du côté du lecteur de la lettre. Ont été ici retenus comme lecteurs le destinaire et, comme lecteurs extérieurs, le tiers, contemporain ou chercheur. La question de l'intention de dire vrai ou faux, de faire littéraire ou non, de vouloir ou d'être indifférent à être publié n'a plus alors vraiment d'importance. La perception que le lecteur a de la lettre est très révélatrice du degré de vérité qu'il lui accorde et que, par conséquent, a cette lettre. Une distinction entre les lecteurs est aussi pertinente: les destinataires ont fortement tendance à considérer comme vraies toutes les lettres qui leur parviennent, alors que le tiers ou le chercheur ont fortement tendance à douter de la vérité de la lettre. Cette différence de perception serait produite par la distance normalement présente chez le tiers. Cependant, dans le cas de l'éditeur ou de l'historien de la littérature, cette distance serait tellement grande qu'elle en viendrait à substituer totalement à l'épistolier le chercheur qui, ainsi, en vient tout à fait à oublier celui à qui la lettre s'adresse avant tout, le lecteur. D'ailleurs la très grande majorité des correspondances éditées ne publient que les lettres envoyées (et par les plus illustres), pratique qui sera probablement encore plus fortement décriée dans quelques années, tellement elle va à l'encontre des fondements épistémologiques de la pratique épistolaire. Le chercheur s'imagine alors être l'auteur de ces lettres. Il les corrige, en restituant les vraies dates, les vrais mots, parfois même il en corrige l'interprétation (c'est le cas, par exemple, dans l'édition de la Correspondance de George Sand par Georges

13 "La vigoureuse critique des travaux de B. Bray par R. Duchêne [...] fait éclater, à propos des Lettres de $\mathrm{M}^{\text {me }}$ de Sévigné, l'antagonisme de deux conceptions du texte littéraire, l'affrontement d'une méthode de stricte interprétation historique et d'une critique d'inspiration "textuelle" ou formaliste", Bernard Beugnot, op. cit., p. 198. 
Lubin ${ }^{14}$ ), et, plus grave, ne nous livre que très rarement le point de vue de celui qui les a lues dans leur plus simple apparat. La publication également, jusqu'ici négligée, des lettres reçues, chaque fois qu'elle serait possible, nous fournirait le point de vue occulté du lecteur. Dans ce sens, l'édition de corpus, dans certains cas plus restreints à cause de leur rareté, mais plus ouverts à la réalité du dialogue épistolaire, donc plus représentatifs, serait à privilégier à la place de grandes correspondances soliloques (v.g. celles de Voltaire, de Sand, de Groulx, etc.), où les "lettres d'auteur" sont présentées comme des unités textuelles autosignifiantes. Encore faut-il que le chercheur choisisse d'en faire l'objet de sa recherche.

La question de l'authenticité de la lettre reste encore une question ontologique (que prétend être celui qui l'écrit?) et morale (et dit-il vrai? est-il honnête?) plutôt qu'une question épistémologique (quel type de connaissance la correspondance produit-elle? et où est-elle produite?). En situant l'objet d'analyse du côté du lecteur, la question esthétique n'est pas pour autant négligée. La "belle" lettre est alors tout simplement celle qui est perçue comme telle, non par celui qui l'écrit, peu importe son intention esthétique, mais bien par celui qui la reçoit et qui est invité à en produire une à son tour. Le jeu de la question et de la réponse et qui serait, selon Jauss ${ }^{15}$, au fondement de la jouissance esthétique, trouve un terrain tout à fait propice dans la pratique épistolaire, laquelle cependant n'a pas encore fait l'objet d'une analyse de réception. C'est l'hypothèse que je voudrais ici vérifier. La connaissance produite par la lettre passe certes par le jeu de la séduction, mais comme la lettre est la seule forme d'écriture qui exige une réponse de son lecteur, la prise en compte de cette réponse est incontournable quelle que soit la perspective d'analyse adoptée. La réponse donnée à la lettre est en effet à ce

14 George Sand, Correspondance, éditée par Georges Lubin, Paris, Garnier, 24 volumes depuis 1964. L'éditeur fait souvent des commentaires pour informer le lecteur de la "bonne" interprétation qu'il devrait donner aux lettres, pas toujours assez véridiques à ses yeux. Dans le tome 5, par exemple, Lubin commente ainsi, en note en bas de page, un passage d'une lettre de la romancière envoyée au poète-maçon Charles Poncy: "Ne commentons pas... On voit bien dans quelle intention (bonne) George Sand commet ce gros mensonge" (p. 754).

15 Hans Robert Jauss, Pour une esthétique de la réception (1968), Paris, Gallimard, coll. "Tel ", 1990, 305 p. Je développerai plus loin cette hypothèse. 
32

point constitutive de cette connaissance, que nous avons curieusement toujours un peu de mal, dans une correspondance générale, à distinguer les lettres reçues des lettres envoyées. À défaut d'en tenir compte, la connaissance, pourtant si riche par sa pluridimensionnalité à cause de grande capacité référentielle, produite par et dans l'échange épistolaire, nous est trop souvent parcellement livrée. Une histoire de la connaissance traduisant cet échange particulier, par exemple la connaissance des représentations esthétiques, reste à faire avec toutes les présumées fausses, insignifiantes, répétitives et inesthétiques lettres qui ont été écartées du corpus épistolaire de référence sans autre explication méthodologique. Finalement, ce qui importe à l'histoire littéraire, c'est de révéler chez les correspondants, tour à tour épistolier et lecteur, "le masque que porte chacun d'eux, et non la vérité cachée derrière le masque" ${ }^{16}$. Or, rien n'est plus révélateur que l'Autre sur l'utilisation et la signification de ce masque.

\section{La fausse lettre, selon les correspondants, est toujours vraie}

Tous ces gens-là trompent sciemment et ne vivent que de tromperies.

(Lettre de Louis-Antoine Dessaulles à sa fille Caroline, 20 septembre $1877^{17}$ )

Les fausses lettres sont un matériau privilégié pour étudier ce qu'on entend par une vraie lettre. Les vraies lettres ne disent pas toujours la vérité, cela est convenu. Certaines la disent plus ou moins; d'autres l'exagèrent et ainsi se rendent moins crédibles. C'est le cas du jeune Arthur Buies qui, contre la volonté paternelle s'est exilé à Paris dans le but louable d'y poursuivre ses études. À dix-sept ans, il écrit à sa grand-tante qui en a la tutelle et qui est en attente des résultats de cette éducation autogérée, une lettre qui n'a pas dû la rassurer par sa véracité:

16 Oscar Wilde, Le déclin du mensonge, Paris, Éditions Complexe, 1986, p. 37.

17 Louis-Antoine Dessaulles, lettre à sa fille Caroline, 20 septembre 1877, dans Un Canadien français en Belgique au $19^{\circ}$ siècle: correspondance d'exil de L.-A. Dessaulles, 1875-1878, éditée par Éliane Gubin et Yvan Lamonde, Bruxelles, Palais des Académies, Commission royale d'histoire, 1991, p. 182. 
Ma chère tante,

Je veux travailler, compter chaque minute de mon temps comme une perle de plus jetée dans le trésor de l'avenir, comme un grain fertile qui produire des épis... J'ai été contraint d'écrire cette lettre pendant la récréation et comme je travaille déjà 13 heures sans relâche, je me sens incapable de renouveler ma besogne pendant mes quelques minutes de loisir ${ }^{18}$

Sur ce ton qui affecte d'être vrai, Buies répondait ainsi judicieusement à la lettre précédente de sa parente qui le conjurait d'être un modèle: "[...] mais souviens-toi bien, Arthur, que maintenant c'est plus que des promesses qu'il faut; c'est des preuves, oui des preuves de bonne conduite en tout application à persévérer, il faut que tes procédés journaliers et futurs, fassent connaître que tu es devenu tout autre; [...]"19. Suivant à la lettre la recommandation de sa correspondante, Buies se transforme ainsi complètement, comme on l'a vu, dans la lettre envoyée et devient un épistolier à la fois averti et converti pour sauver les apparences. Or, en disant trop la vérité attendue, la lettre de l'étudiant faussement appliqué accuse un décalage qui a même dû provoquer plus d'inquiétude et de colère que d'apaisement chez sa correspondante. Seul, Buies joue ici au maximum le jeu épistolaire.

La typologie de la fausse lettre est assez complexe. Sur une échelle bien graduée, on trouve à un extrême les lettres entièrement inventées (les vraies fausses lettres, telles les Lettres portugaises), tandis qu'à l'autre se situent les lettres tout à fait véridiques (telles les lettres d'affaires). Entre les deux, les lettres amicales ou familiales; surtout du côté des vraies lettres, les lettres d'amour; et plutôt parmi les fausses lettres, les lettres trop charmantes ou raisonnantes. Les lettres familières sont assez spontanément considérées comme vraies, probablement à cause des marques de réalisme qu'elles recèlent au même titre d'ailleurs que celles produisant l'effet de réel dans le roman dit réaliste: individualisation et caractérisation des personnages avec présentation détaillée de leur environnement en temps et espaces réels ${ }^{20}$. En revanche, les lettres argumentatives sèmeraient davan-

18 Arthur Buies, lettre à [Luce G. Drapeau-Casault], 25 novembre 1857, dans Arthur Buies, Correspondance, 1855-1901, édition de Francis Parmentier, Montréal, Guérin, 1993, p. 41.

19 Luce G. Casault, lettre à Arthur Buies, 23 octobre 1857, ibid., p. 40-41.

20 Il s'agirait, selon Ian Watt, des deux principales caractéristiques du réalisme dans le roman moderne. Watt associe ces nouveaux procédés romanesques à 
tage le doute, car, selon Parret, qui analyse la fausse dichotomie entre la rationalité narrative et la rationalité argumentative: "[...] il existe l'intuition chez tous que l'acte de raconter est plus authentique, plus "moral", plus proche de la véritable nature humaine que l'acte de raisonner d'emblée plus manipulatoire, plus "logique" "21. Il n'existe évidemment pas de traité épistolaire où les modèles de lettres seraient classés en fonction de leur degré d'authenticité, de véracité... Toutes les lettres prétendent être vraies, sinon pourquoi les écrirait-on et les lirait-on aussi sérieusement?

La jouissance créée par la lettre n'est d'abord pas esthétique au sens traditionnel ${ }^{22}$; elle relève du plaisir que le lecteur a à la dé-couvrir ${ }^{23}$. Le lecteur prend plaisir à découvrir une vérité à la lettre. Cette découverte est la source de sa satisfaction. La philosophie du langage explique ainsi l'origine de cette satisfaction: "La notion de satisfaction d'un acte de discours est basée sur celle de vérité par correspondance. [...] Cependant, [...] il ne suffit pas, pour qu'un acte de discours soit satisfait, qu'il y ait ajustement ou correspondance entre son contenu propositionnel et le monde. Il faut aussi que cette correspondance soit établie suivant la direction d'ajustement propre à son but illocutoire" 24 . D'où l'importance, pour statuer de la véridicité de la lettre de tenir compte des conditions de production de la réception de la lettre.

la philosophie cartésienne qui défend le principe "selon lequel la recherche de la vérité est conçue comme une matière absolument individuelle" ("Réalisme et forme romanesque", dans Roland Barthes et al., Littérature et réalité, Paris, Seuil, coll. " Points ", 1982, p. 16, 23).

21 Herman Parret, op. cit., p. 49-50.

22 L'esthétique romantique d'origine hégélienne, dont la modernité est encore très tributaire, consiste à établir une adéquation absolue entre le beau et l'art : "Pour Hegel, seul l'art peut être dit beau, parce que la beauté est d'essence spirituelle." (Marc Sherringham, Introduction à la philosopbie estbétique, Paris, Petite bibliothèque Payot, 1992, p. 226). Kant avait cependant proposé une esthétique plus ouverte sur le monde. Il "instaure le second paradigme de l'esthétique philosophique sur la substitution du sujet à l'être. Ce faisant, il installe l'esthêtique au plus intime du sujet comme le sentiment de soi qu'éprouve la subjectivité dans sa liberté. Le beau objectif peut être alors indifféremment naturel ou artistique, puisqu'il n'est plus que l'occasion, plus ou moins favorable, pour le sujet d'éprouver la finalité interne de ses propres facultés" (ibid., p. 225). La réflexion proposée ici s'inscrit dans le paradigme kantien.

23 Manon Brunet, "L'intimité de la lettre au $19^{\mathrm{e}}$ siècle: de la lettre cachetée à la lettre ouverte ", dans Manon Brunet, Serge Gagnon (dir.), op. cit., p. 131-148.

24 Daniel Vanderveken, Les actes du discours: essai de philosophie du langage et de l'esprit sur la signification des énonciations, Liège/Bruxelles, Pierre Mardaga Éditeur, 1988, p. 134. 
Le pacte d'intimité, tel que l'appelle Vincent Kaufmann ${ }^{25}$, désigne alors la relation privilégiée entretenue, à distance, entre l'auteur et le lecteur de la lettre. Kaufmann affirme même que cette distance est désirée, puisqu'elle est nécessaire à l'accomplissement de l'œuvre littéraire. La correspondance serait "le fameux chaînon manquant entre l'homme et l'œuvre" 26 . Ainsi, les lettres écrites à son amante Louise au cours de la rédaction de Madame Bovary, dénotent la nécessité pour Flaubert de "faire le vide en lui et autour de lui, et [de] se mettre ainsi à distance de tout ${ }^{27}$. Au départ donc, la correspondance apparaît comme un acte faux, manqué. Voulant réduire la distance entre l'épistolier et l'objet de son désir, elle ne fait, en somme, que l'accroître si elle est entretenue: "Si l'écrivain voulait communiquer, il n'écrirait pas, et cette possibilité idéale de ne pas communiquer est sans doute la raison pour laquelle il entretient souvent des correspondances volumineuses, acharnées, s'efforçant inlassablement de convoquer autrui pour mieux le révoquer" 28 . Cette équivoque épistolaire est particulièrement bien formulée par Alain Grandbois dans une de ses lettres à Lucienne qui commence ainsi : "Ta lettre me fait regretter d'être allé te voir "29 et qui se termine sur un appel à la distance commandé paradoxalement par le grand amour (véritable, se demandera-t-on?): "J'ai peur maintenant de nous revoir. Encore se déchirer. Comme si nous étions des ennemis. Je t'aime". Le déchirement est à la fois celui du sentiment et du papier, puisqu'il s'agit d'une des dernières lettres envoyées à Lucienne.

Quoiqu'on puisse faire dire éventuellement à la lettre sur le plan biographique ou historique, écrire ou lire une lettre est d'abord et avant tout un acte érotique. Cette fonction du genre sert d'ailleurs magnifiquement bien le sentiment amoureux. Les chefsd'œuvre de la littérature épistolaire, tels les Lettres portugaises (1669) et Les liaisons dangereuses (1782) de Laclos, en ont largement usé. La lettre est la seule forme d'écriture qui exige une réponse du lecteur. Le jeu de la séduction est-il vrai ou faux? C'est précisément ce jeu, plus ou moins joué, ouvert, qui nous laisse croire que la lettre est plus ou moins fausse. Pourtant "La logique

\footnotetext{
25 Vincent Kaufmann, L'équivoque épistolaire, op. cit., p. 159.

26 Ibid., p. 9.

27 Ibid., p. 28.

28 Ibid., p. 8.

29 Alain Grandbois, lettre à Lucienne, [23 septembre 1933], dans Alain Grandbois, Lettres à Lucienne, op. cit., p. 198.
} 
de la séduction est une parodie. [...] le séducteur n'est pas un menteur. Il n'est pas un manipulateur. Le séducteur n'est pas non sincère, la séduction n'est pas un malheur. [...] Le séducteur est un esthète, un contemplateur de la beauté, un enthousiaste. En fait, ce n'est que l'enthousiasme qui séduit " ${ }^{30}$. La "tentation pour une écriture de l'intime " 31 serait-elle l'indice de l'authenticité ou de l'inauthenticité de l'acte et de la relation épistolaires? Dans ce jeu esthétique, la lettre ne sert pas vraiment à exprimer le Moi, mais plutôt le désir de participer et de voir l'autre participer à ce jeu de la révélation. Comme l'observe fort justement Hélène Védrine à partir du cas de la correspondance du graveur belge de la fin du $19^{\mathrm{e}}$ siècle, Félicien Rops, lequel a une très grande conscience de jouer (à) ce jeu: "La lettre ne sert pas à exprimer quelque chose de personnel, mais sert à dire que l'on est dans le désir de l'exprimer " 32 .

Une étude approfondie de la terminologie dont se servent les correspondants pour décrire l'acte épistolaire se faisant, qu'il s'agisse de lettres banales, amoureuses ou savantes, montrerait à quel point le jeu de la séduction y est présent. Par exemple, il est un lieu commun de s'épancher, au début d'une lettre, du plaisir qu'on a éprouvé à la réception de celle de l'autre. Ce plaisir est intimement associé au degré de vérité que le correspondant accorde à la lettre reçue: "Ta dernière lettre, comme toujours, m'a causé un vif plaisir. On y sent l'accent du coeur et de l'affection vraie, 33 . Il en est de même du thème de l'attente épistolaire. Plus l'attente est longue, plus le destinataire est tourmenté à propos de l'authenticité de sa relation avec l'autre, de la vérité de ses belles paroles, que cette relation soit amoureuse ou non: "J'ai eu, cher fils, un moment de satisfaction en revenant de la messe, ayant trouvé une lettre de ta sour dans laquelle j'en ai trouvé une de toi. Quelle joie pour moi en voyant cette écriture! Mais, en voyant la date, j'ai été désolée! Quoi, il faut six mois pour avoir de tes nouvelles? Pense donc, aimable fils, ce que peut souffrir ta mère [... $]_{n} 34$.

30 Herman Parret, op. cit., p. 89.

31 Vincent Kaufmann, op. cit., p. 158.

32 Hélène Védrine, “Lettre, texte et image : la représentation dans la correspondance de Félicien Rops", dans Mireille Bossis (dir.), La lettre à la croisée de l'individuel et du social, Paris, Kimé, 1994, p. 167.

33 Gabrielle Roy, lettre à sa sour Bernadette, 5 janvier 1966, dans Gabrielle Roy, Ma chère petite sour : lettres à Bemadette, 1943-1970, op. cit., p. 113.

34 Élisabeth Bégon, lettre à son gendre, 2 juin 1750, dans Élisabeth Bégon, Lettres au cher fils : correspondance d'Élisabeth Bégon avec son gendre, 1748- 
Quand les correspondants se transforment en retour en épistoliers, ils témoignent d'une conscience aiguë de la nécessité de paraître vrais: "Je t'ai écrit deux ou trois lettres, que j'ai par la suite déchirées. Elles rendaient un son faux. La vérité est que je ne sais plus t'écrire. Il faudrait faire un choix, dire ceci ou cela. Ou tout dire. Je ne le peux pas. Trop de choses se pressent en moi, oscillant entre deux pôles, et qui se heurtent, se contredisent. Je te crie "Reviens, reviens", et je te crie "Je ne veux plus te revoir jamais". Il n'y a pas de vérité au milieu"35. Grandbois essaie ici d'expliquer le paradoxe de la distance épistolaire à la fois nécessaire et douloureuse. Mais plus la distance physique est grande entre les correspondants, plus la lettre risque d'être considérée comme fausse, car, pourrait-on dire, "a beau mentir qui écrit de loin". Louis-Antoine Dessaulles, exprime très bien ce malaise, dans ses lettres envoyées à sa fille, durant son exil en Belgique de 1875 à 1878: "Il m'arrive des choses si étranges que je crains presque de les dire. Il me semble que vous allez croire ou à des exagérations ou à des erreurs de ma part envers les autres. D'un autre côté, tu ne dois pas me croire capable de te tromper, toi que j'aime par dessus tout " 36 . Le passage du "vous" au "tu" est particulièrement éloquent sur le choix fait par Dessaulles du destinataire familial qui saura le mieux découvrir la vérité dans sa lettre.

Depuis le siècle précieux de Madame de Sévigné, de Guilleragues et de La princesse de Clèves (1671), les traités d'art épistolaire ont toujours cherché à nous instruire sur la nature de ce jeu. Si l'auteur de la lettre doit tenir compte du type de relation entretenue avec son correspondant pour adapter le contenu de sa révélation au ton devant être adopté en chacune des circonstances de la vie ${ }^{37}$, c'est qu'on "soupçonne de peu de sincérité tout

1753, présentée par Nicole Deschamps, Montréal, Hurtubise HMH, 1972, p. 181 .

35 Alain Grandbois, lettre à Lucienne, [13 novembre 1932], op. cit., p. 76.

36 Louis-Antoine Dessaulles, lettre à sa fille Caroline, 6 avril 1877, op. cit., p. 43.

37 Manon Brunet, "Les traités d'art épistolaire au $19^{\mathrm{e}}$ siècle québécois : rhétorique et code social ", dans Benoît Melançon, Pierre Popovic (dir.), Les facultés de lettres: recherches récentes sur l'épistolaire français et québécois, Montréal, Département d'études françaises, 1993, p. 45-72 ; Cécile Dauphin, "Les manuels épistolaires au $19^{\mathrm{e}}$ siècle", dans Roger Chartier (dir.), La correspondance: les usages de la lettre au $19^{\circ}$ siècle, Paris, Fayard, 1991, p. 209272 . 
ce qui n'imite pas ingénieusement la nature " ${ }^{38}$. Or, selon ces traités, la nature est la seule motivation qui devrait guider l'auteur d'une lettre sincère. Le naturel bien inspiré est opposé au travail épistolaire dont la finalité est douteuse, "la vérité seule étant d'une force merveilleuse pour tourner l'esprit où elle veut "39. Dans cette perspective, l'esthétique, la littérarité joue contre la sincérité. Est-ce nécessaire et vraisemblable? Je voudrais attirer l'attention, à partir d'une analyse de la réception épistolaire, sur la fonction esthétique de la lettre qui est récupérable dans tous les cas où la lettre procure une jouissance tirée du jeu de la séduction dont elle n'est que l'instrument. Ce jeu peut être jugé comme plus ou moins "subtil", plus ou moins esthétique. Cette considération nous amène cependant plus sur le terrain du jugement que de la jouissance esthétique. L'un ne va probablement pas sans l'autre, mais l'important est que le jeu joue. Or, il semble que ce soit généralement le cas, si l'on se fie au nombre particulièrement élevé de lettres qui répondent à l'exigence d'une réponse.

Lorsqu'on qualifie de vraie ou de fausse une lettre, c'est en réalité qu'on découvre, placé à l'extérieur de la relation épistolaire le plus souvent, la capacité de la lettre à cacher plus ou moins implicitement ce jeu constant entre la distance et le rapprochement désirés. Mais il est souvent difficile d'évaluer très exactement l'authenticité de ce jeu. Le degré de complicité exprimée entre les correspondants peut servir d'indice. Mais souvent il s'avère insuffisant pour juger de l'authenticité de la lettre lorsque celle-ci semble trop travaillée pour exprimer des sentiments d'ordinaire trop naturels. La lettre amoureuse est le genre épistolaire qui pose de manière le plus aiguë ce problème, puisqu'elle sert à exprimer un très fort désir de rapprochement. La poésie utilisée pour l'exprimer peut alors mettre sérieusement en doute la véracité des sentiments exprimés, aux yeux du lecteur extérieur.

C'est pourquoi la lettre amoureuse a deux statuts de vérité, selon que l'amoureux est plus ou moins doué de la parole,

38 César Pierre Richelet, Les plus belles lettres françoises sur toutes sortes de sujets tirées des meilleurs auteurs, avec des notes, La Haye, Meyndert Vytwerf/Louis et Henri van Dole, 1699, tome 1, p. 29.

39 Antoine Courtin, Nouveau traité de la civilité qui se pratique en France parmi les bonnêtes gens, augmenté de la civilité chrétienne, Bruxelles, François Foppens, 1708, p. 149. 
parole exercée par une formation courante ou professionnelle à l'écriture. Ainsi, s'il apparaît tout à fait vraisemblable lorsque Ginette affirme à Vincent: "[...] je t'aime, je t'aime, je t'aime, gros, gros, gros. Il faudrait que je trouve autre chose à dire mais quoi " 40 , que Ginette aime effectivement passionnément Vincent, il est plus difficile de croire en l'amour passionné qu'Alain Grandbois ressent vis-à-vis de Lucienne quand il s'exprime à la manière du poète: "Tu es seule aussi dans ma pensée, et au centre de mon cour. Mais tu me fais souffrir comme si vous étiez mille. [...] J'imagine que je n'étais fait que pour flâner, rire, goûter les heures les unes après les autres, auprès d'une femme aimée. La femme aimée, c'est toi. [...] Et toi, où es-tu, que fais-tu? Il est onze heures. Le monde est mort. [...] mes mains tendent inutilement vers toi. Mes mains sont vides. Le bonheur ne m'aime pas " ${ }^{41}$. La lettre d'amour est cependant toujours considérée comme vraie par le correspondant. En publiant cette lettre, il est à la fois surprenant et intéressant de voir que Lucienne ne peut s'empêcher de se remémorer à haute voix, dans une note en bas de page, ce sentiment vivement ressenti de l'authenticité de la lettre, de la garantie affective de la relation que la lettre du poète évoquait et représentait alors pour elle: "Ce sont des affirmations de ce genre auxquelles je m'accrochais pour croire à son amour " ${ }^{42}$. Chaque fois donc qu'il y a un doute sur la sincérité de la lettre, il faut avouer qu'il s'agit d'une interprétation donnée à la lettre par un tiers ou par un correspondant qui est devenu un tiers, une fois la relation privilégiée estompée ou définitivement éteinte.

Ainsi, convenons que les lettres, peu importe leur contenu, sont toujours d'abord prises au sérieux, c'est-à-dire considérées comme vraies par ceux qui les reçoivent. La trop grande distance émotive, physique et temporelle amène soit l'ex-correspondant, soit l'intrus ou soit le chercheur complètement au dehors de cette relation, à donner une nouvelle interprétation à la lettre. Par un retournement prévisible, la lettre, qui jouait dangereusement le jeu du rapprochement et de la mise à distance, finit toujours, si

40 Ginette, lettre à Vincent, 21 juillet 1964, citée par Roch Hurtubise, op. cit., p. 157.

41 Alain Grandbois, lettre à Lucienne, [25 novembre 1932], op. cit., p. 114-115.

42 Note de Lucienne sur la lettre d'Alain Grandbois à Lucienne, [25 novembre 1932], ibid., p. 115 , note 1 . 
40

elle s'allonge, par créer la distance. D'où les recommandations parfois faites de brûler ou de garder les lettres en prévision de ce renversement pénible de situation: "Garde aussi la lettre qu'elle t'a envoyée pour le cas où elle deviendrait encore plus dangereuse. Elle a bien eu, elle, la méchanceté d'insérer dans ce fameux manuscrit une lettre intime de moi à elle, la seule où je lui avais un peu ouvert mon cœur ${ }^{43}$. Ces lettres perdues, caviardées ou tronquées sont appelées tantôt "lettres-fantômes" 44 , tantôt lettres attestées. Les justifications imprécises quant à leur absence éveillent la curiosité spontanée pour l'objet intime caché et leur est accordée une potentialité de vérité beaucoup plus élevée que les lettres volontairement conservées, car "La lettre brûlée est la figure ultime du secret ${ }^{45}$. Une exception vient cependant confirmer la règle d'interprétation habituellement réservée aux seules lettres perdues: il s'agit des lettres qui ont été précieusement gardées, mais malgré toutes les recommandations de destruction et malgré leur haut degré de confidentialité confirmé par le libellé "Confidentielle" apparaissant sur la lettre. Celles-là aussi sont considérées, vues de l'extérieur toujours, comme faisant partie des plus authentiques, parce qu'étant tenues des plus secrètes. La question demeure cependant: pourquoi ce qui est caché serait-il plus vrai que ce qui est dit publiquement? Tout ceci serait l'effet d'une pure illusion empirique. Pour l'affirmer, Parret s'en remet à Wittgenstein qui nous ramène au critère d'authenticité: "La certitude concernant les faits et les événements de ma vie n'existe qu'au niveau d'une sanction émanant de la communauté dans laquelle je me construis. Je peux évidemment imaginer qu'il y a des domaines qui me restent personnels et qui sont de l'ordre de mon secret personnel. Mais même le secret doit être sanctionné comme tel, sans quoi il n'aurait aucun attrait ou force séductrice ${ }^{46}$.

43 Gabrielle Roy, lettre à sa sœur Bernadette, 24 mai 1969, op. cit., p. 156.

44 Bernard Chassé, "Sur quelques lettres-fantômes. Genèse d'une édition critique de la correspondance d'Alain Grandbois », dans Benoît Melançon, Pierre Popovic (dir.), op. cit., p. 193.

45 Michelle Perrot, "Le secret de la correspondance au $19^{\mathrm{e}}$ siècle ", dans Mireille Bossis (dir.), L'épistolarité à travers les siècles: geste de communication et/ou d'écriture, Stuttgart, Franz Steiner Verlag, 1990, p. 188. Herman Parret, op. cit., p. 40-41. 


\section{La fausse lettre, selon les autres, est toujours celle des autres}

[...] le potentiel de vérité et l'exigence de vérité ne sont explicables que si nous recourons à l'interdépendance complexe entre les différentes dimensions de la vérité, telle qu'elle existe dans l'expérience d'une vie que dans la formation ou dans la transformation d'attitudes, de modes de perception et d'interprétations. (Albrecht Wellmer ${ }^{47}$ )

Le 18 août 1883, Alfred Garneau, fils de l'historien national, écrit à Casgrain: "Je veux aussi vous dire en confidence que l'Académie française va probablement couronner l'ouvrage de mon père: M. Marmier me l'a presque assuré. [...] Ne parlez point de cela " ${ }^{48}$. Dix jours plus tard, Casgrain s'empresse d'écrire à son ami Joseph Marmette, beau-frère d'Alfred Garneau: "Il [Garneau] me dit en confidence que $M$. Marmier lui a assuré qu'il allait obtenir un grand prix d'histoire pour l'œuvre de son père " 49. Comment interpréter cette confidence en fonction du critère d'authenticité? Deux interprétations sont possibles, selon que l'on s'identifie à l'épistolier (le destinateur) ou à celui qui reçoit la lettre, la lit (le destinataire). La différence d'interprétation peut être énorme.

En lisant la lettre de Casgrain à Marmette, le chercheur va avoir le réflexe de douter sérieusement de la réelle complicité entre Casgrain et Garneau. Pourquoi? Le lecteur extérieur s'identifie d'abord au premier épistolier, Alfred Garneau. Il est vraiment assuré que Garneau fait une réelle confidence à son très grand ami Casgrain puisqu'il prend la peine non seulement de lui écrire "Je veux aussi vous dire en confidence [...]", mais aussi d'ajouter en insistant: "Ne parlez point de cela". Lorsque Casgrain devient

47 Albrecht Wellmer, "Vérité, apparence, réconciliation: Adorno et le sauvetage esthétique de la modernité ", Rainer Rochlitz (éd.), Théories estbétiques après Adorno, Paris, Actes Sud, 1990, p. 279.

48 Alfred Garneau, lettre à Henri Raymond Casgrain, 18 août 1883, ASQ, Lettres diverses, tome $10(\mathrm{O} 457), \mathrm{n}^{\circ} 84$.

49 Henri Raymond Casgrain, lettre à Joseph Marmette, 28 août 1883, Université Laval, Fonds Brodeur, 209.1/25/7/104. 
42

à son tour auteur d'une lettre, en écrivant à son ami de longue date, Joseph Marmette, le lecteur extérieur s'identifie au nouvel épistolier. Il imagine le contenu de la lettre qu'il aurait pu écrire à Marmette dans les circonstances énoncées par le premier épistolier, Garneau, c'est-à-dire celles clairement exprimées de la confidence. La lettre que le lecteur extérieur aurait écrite à Marmette aurait été entièrement différente de celle véritablement écrite par Casgrain, d'où l'étonnement et d'où le jugement portê sur le statut de vérité de la lettre casgrainienne. Respectant en tout point la demande de confidentialité exprimée par Garneau dans la première lettre, le lecteur extérieur n'aurait surtout pas eu ni la naïveté ni l'affront d'écrire à Marmette que Garneau "me dit en confidence que». Le deuxième épistolier (Casgrain) apparaît alors comme un traître du premier (Garneau), étant donné qu'il a très consciencieusement brisé le pacte amical en dénonçant le pacte épistolaire scellé sous le couvert de la confidentialité, donc de la révélation unique.

Or, cette interprétation fabriquée par le lecteur extérieur pourrait s'avérer très différente de celle qui a pu être alors effective au moment où la correspondance échangée eut lieu. Cette interprétation effective peut être accessible au lecteur extérieur s'il opère un renversement complet de son lieu d'observation privilégié qui, jusqu'ici, était celui de l'auteur de la lettre. L'auteur et sa pratique comme lieu d'observation peut suffire, épistémologiquement parlant, à l'interprétation romanesque, par exemple ; d'où le réflexe du chercheur à s'identifier à l'auteur de la lettre. Toutefois il est nettement insuffisant dans le cas de la lettre parce qu'il éclipse entièrement l'important rôle joué par celui à qui la lettre s'adresse obligatoirement, c'est-à-dire celui qui reçoit la lettre et de qui une réponse est exigée. En s'identifiant, cette fois, au véritable lecteur de la lettre, l'observateur extérieur risque d'être potentiellement plus près de la "vérité" inventée pour le plaisir et la nécessité du jeu social.

Reprenons le scénario sous cet angle du lecteur. Casgrain reçoit la lettre de Garneau qui l'invite à être très discret sur la bonne nouvelle communiquée concernant l'important appui que représente l'académicien Xavier Marmier en vue de l'obtention d'un prix de l'Académie française en faveur de l'ouvrage historique de son défunt père. L'analyse de la réception de cette lettre par Casgrain peut être faite en fonction de son borizon d'attente social, concept utilisé par Jauss et auquel j'ai déjà eu l'occasion 
d'apporter quelques nuances afin de proposer une esthétique de la production de la réception ${ }^{50}$. La prise en compte des conditions de production de la réception, plutôt que de celles de la réception elle-même qui n'est d'ailleurs pas toujours connue (voir supra, les lettres-fantômes), permet de délimiter au moins, à défaut d'une vérité historique assurée qui restera toujours inaccessible, un ensemble fini de lectures possibles de la lettre. Si cette approche a pu être utilisée avec profit dans l'analyse herméneutique et sémantique tant du roman, du théâtre que de la poésie ${ }^{51}$ alors que le lecteur est une personna incognita et qui donne rarement signe de vie, je serais porté à croire qu'elle conviendrait encore davantage, avec tous les ajustements proposés, au genre épistolaire qui, rappelons-le, est la seule forme d'écriture qui exige une réponse de son lecteur et dont la réception du lecteur est plus aisément repérable. La mise au jour de l'horizon d'attente social de la lettre devrait alors se faire plus aisément ou du moins devrait, dû aux conditions épistémologiques de production de sens du genre, obligatoirement prendre en considération l'horizon d'attente possible du destinataire. Tentons de le voir dans le cas où Casgrain reçoit la lettre de Garneau, et Marmette, celle de Casgrain.

Quel sens Casgrain peut-il donner à cette lettre venant de son ami Alfred Garneau? Voyons quelles sont, pour Casgrain, les conditions de lecture de cette lettre. En tant qu'ami intime de Garneau, et même du défunt père de son ami (n'a-t-il pas été appelé par la famille à donner l'extrême-onction en 1866 à FrançoisXavier Garneau?), Casgrain s'attend à être mis dans la confidence de tout ce qui concerne de près l'historien national et son œuvre. $\mathrm{Au}$ moment où il reçoit cette lettre, il a déjà été sollicité par Alfred Garneau pour écrire une critique avisée qui réhabiliterait complètement l'historien trop libéral auprès des autorités ecclésiastiques. Cet article, qui paraîtra dans L'opinion publique le 15 novembre 1883 sous le titre de "Histoire du Canada", était très attendu au sein du réseau casgrainien, comme en fera foi cette lettre de Benjamin Sulte à Louis Fréchette débutant par un significatif "Entre nous": "La position va se dessiner! Les prêtres de

50 Manon Brunet, "Pour une esthétique de la production de la réception", Études françaises, vol. XIX, n 3 , hiver 1983-1984, p. 65-82.

51 Hans Robert Jauss, Pour une estbétique de la réception (1978), Paris, Gallimard, 1990, 305 p.; Pour une berméneutique littéraire (1982), Paris, Gallimard, 1988, $457 \mathrm{p}$. 
Laval ne trouvent rien à redire à Garneau dans l'affaire LavalJésuites. Casgrain publiera bientôt un article très fort sur ce sujet dans l'Opinion publique "52. En plus donc de s'attendre à être mis en confidence au sujet de François-Xavier Garneau, Casgrain ne peut pas être surpris outre-mesure du contenu même de la confidence. En effet, il s'attendait également à ce qu'Alfred Garneau, de retour de son premier voyage en Europe en 1883, lui donnât des nouvelles de sa rencontre avec une de ses propres connaissances françaises, Xavier Marmier, lequel deviendra un ami au cours des fréquents voyages de recherche de Casgrain en Europe.

Analysons maintenant la lecture probable de Joseph Marmette à la réception de la lettre de Casgrain. À quoi Marmette peut-il vraisemblablement s'attendre d'une lettre de Casgrain lui étant envoyée à ce moment-là? Joseph Marmette se trouve alors à Paris en mission de recherche depuis un an. Il est très fier d'être le gendre de l'historien national, de par son mariage avec Joséphine Garneau, ce qui en fait donc le beau-frère d'Alfred. Or, durant son séjour parisien, Marmette était devenu un ami proche de Marmier. Voici d'ailleurs comment il relate dans ses Récits et souvenirs sa première rencontre avec l'académicien: "Muni d'une lettre d'introduction que je devais à l'obligeance de mon ami Faucher de St-Maurice, je me présentais, dès les premiers jours de mon arrivée à Paris, chez l'éminent académicien" "53. Et voilà comment l'auteur des Lettres sur l'Amérique ${ }^{54}$ le reçoit: "Ah! vous avez épousé la fille de $M$. Garneau, me dit-il en lisant la lettre que je lui avais donnée; c'était un écrivain bien distingué, un excellent homme, que je me fais gloire d'avoir connu au Canada " 55 . Marmette arrive donc en pays de connaissance et

52 Benjamin Sulte, lettre à [Louis Fréchettel, 6 novembre 1883, ANC, Fonds Fréchette, MG 29D40, volume 5, p. 3840.

53 Joseph Marmette, Récits et souvenirs, Montréal, Librairie Beauchemin, 1925, p. 133 .

54 Dans le récit de son voyage fait au Canada en 1849, Marmier fait mention de sa rencontre avec l'historien national: "M. Garneau a eu la bonté de m'accompagner avec son excellent concitoyen $M$. Faribault sur un champ de bataille qui me rappelait de meilleurs souvenirs que celui des Plaines d'Abraham [il s'agit de Sainte-Foy, près des chutes Montmorency, lieu où Lévis livra la dernière bataille avant la conquête anglaise]" (Lettres sur l'Amérique, Paris, Arthus Bertrand, [1851], tome 1, p. 173). Il ajoute aussi plus loin un commentaire sur l'œuvre de Garneau: "Mais Québec a de plus que Montréal quelques poëtes, un historien d'un grand mérite, M. Garneau [...]" (p. 177).

55 Joseph Marmette, op. cit., p. 133. 
potentiellement de reconnaissance institutionnelle ("Aussi suis-je tout heureux de posséder un exemplaire de sa belle histoire", ajoute Marmier ${ }^{56}$ ), puisque François-Xavier Garneau était un des premiers amis canadiens de l'éminent académicien.

Ce récit de la première rencontre nous apprend aussi quelque chose d'essentiel pour la compréhension des conditions de réception de la lettre de Casgrain envoyée à Marmette. Marmette aurait utilisé une lettre de Faucher de Saint-Maurice, et non de Casgrain ni d'Alfred Garneau notamment, pour être introduit auprès de Marmier. Ce qui laisse supposer que Marmette aurait été le premier du triumvirat canadien dont il est ici question (Garneau-Casgrain-Marmette) à avoir rencontré Marmier. Ainsi, quand son beau-frère Alfred arrive à Paris à l'été 1883, Marmette est non seulement là pour l'accueillir, mais aussi pour l'introduire auprès de Marmier, dans le but, on l'imagine bien, de continuer à faire avancer la cause qu'il a déjà discrètement commencé à plaider en faveur de l'attribution d'un prix d'histoire par l'Académie française à l'ouvrage de son beau-père, l'Histoire du Canada. Une lettre d'Alfred Garneau, envoyée de Paris le 13 juillet 1883 à sa femme Élodie Globensky, confirme la présence dle Marmette au moment où Marmier "a presque assuré " à Garneau que "l'Académie française va probablement couronner l'ouvrage de [son] père ": "J'ai fait visite aujourd'hui à M. Marmier avec Joseph [Marmette ] et Jos. [...] Il veut m'obtenir une distinction de l'Académie - un prix de deux ou trois mille francs! [...] Il se croit sûr de réussir. [...] Je te dis cela en toute confidence, n'en parle à personne autre que tes sours. Mon indiscrétion serait inexcusable, 57 .

Quand Marmette reçoit la lettre de Casgrain, il est donc déjà parfaitement dans la confidence, puisqu'il se trouvait avec Garneau chez Marmier le jour mémorable et parce qu'en plus il avait été le principal instigateur de l'affaire en terrain français. Casgrain sachant très bien cela, ne fait alors aucune confidence que Marmette ne connaisse déjà et, par conséquent, Marmette lit sa lettre tout simplement comme une preuve de reconnaissance, une sorte de remerciement amical et profond du père de la littérature nationale qui l'a toujours appuyé dans ses initiatives et qui se réjouit

57 Alfred Garneau, lettre à Élodie Globensky, 13 juillet 1883, Famille Garneau. 
de toutes les démarches que Marmette et Garneau ont entreprises dans le but de faire avancer, à l'étranger, la cause de Garneau et celle de la littérature canadienne toute entière. D'ailleurs, Marmette pouvait très bien lire ce sentiment de fierté dans la suite de la lettre de Casgrain: "Plus il y aura d'ouvrages canadiens couronnés, plus nous affirmerons notre supériorité sur les Anglais, du moins en matière d'intelligence " 58 . Contrairement à l'interprétation donnée par le lecteur extérieur identifié à l'épistolier, Casgrain est ici le dernier et non le premier à être mis dans la confidence. Casgrain n'est plus perçu comme un "traître", sa lettre rend compte de ce qu'il y a de plus authentiquement vécu entre les trois hommes de lettres et amis qui oeuvrent, en parfaite complicité et discrétion, à la reconnaissance étrangère de la littérature canadienne. Pour ne perdre aucune chance auprès de l'Académie, Marmier, Garneau, Casgrain et Marmette conviennent donc de garder secret le résultat de leurs stratégies littéraires, autrement cela "serait inexcusable". C'est pourquoi quand Casgrain écrit à Marmette : "Il [Garneau] me dit en confidence que [...]", Marmette lit aisément: "Casgrain m'assure qu'il va garder pour lui la confidence que Garneau et moi lui faisons que [...]".

On voit donc la nette différence d'interprétation de cette lettre de Casgrain à Marmette que peut faire le lecteur extérieur, extérieur aux conditions de production de la correspondance et à la complexité du fonctionnement du réseau littéraire dont l'échange des lettres témoigne, selon qu'il observe soit celui qui écrit la lettre (l'épistolier), soit celui qui la reçoit, qui la lit, qui l'interprète (le destinataire). Faisant fi des conditions de production de réception particulières de la lettre, la tendance est à analyser la lettre comme un roman, à cause de la similitude de son effet de réel, et à découvrir l'intentionalité de l'auteur afin d'évaluer l'authenticité du texte. Or, l'écriture de la lettre a été, à mon avis, jusqu'à maintenant suranalysée par rapport à sa lecture. Pourtant, comme nous l'avons vu, l'analyse de l'horizon d'attente social éclaire autant, sinon plus, la signification réelle de la lettre écrite pour être lue, selon des paramètres pluriels mais relativement bien circonstanciés pour pouvoir en recueillir au moins les plus essentiels à la compréhension de ce que la lettre pouvait vouloir dire dans le jeu de la séduction. L'exemple apporté par la lettre de Casgrain montre que l'authenticité, la vérité de la lettre

58 Henri Raymond Casgrain, lettre à Joseph Marmette, 28 août 1883, op. cit. 
est indissociable du sens, de la réception que son véritable destinataire lui donne en toute connaissance de cause.

Il n'existe pas en réalité de fausses lettres, ni de par leur contenu, ni de par l'interprétation qui peut être faite de leur contenu par le correspondant destinataire. Garneau, Casgrain, Marmette s'écrivent de vraies lettres et les lisent comme tel. Tant que ces lettres sont vraiment écrites et lues par ceux qui s'en prétendent les auteurs et lecteurs, elles sont vraies. Elles peuvent dire des mensonges, mais l'historien ne pourra jamais arriver à en mesurer l'authenticité en fonction des mensonges, apparents ou non, évidents ou non, qui y figurent. Les véritables intentions des épistoliers sont insondables, malgré le nombre et la pertinence des lettres retrouvées. C'est pourquoi, l'historien de la littérature ne peut que se fier à ce que les uns et les autres considèrent alors comme vrai ou faux, puisque c'est ainsi qu'ils vivent et expriment leur propre réalité. La manière choisie pour en rendre compte est évidemment très révélatrice de l'importance accordée à telle vérité ou à telle autre, au même titre qu'elle est révélatrice de la place que chacun occupe au sein du réseau épistolaire, place, formelle ou informelle, qui lui fait voir la réalité d'une façon plutôt que d'une autre. En vérité, seul, celui ou celle du réseau qui lit la lettre peut parvenir de l'intérieur à distinguer avec une vraisemblance certaine, le vrai du faux.

\section{Pour une épistémologie appliquée de l'épistolarité}

Dans son grand souci d'objectivité, la perception que le chercheur a, par exemple de la confidentialité de la lettre, a tendance à être très romantique et mécanique, voire idéaliste et, par conséquent, moralisatrice. Cette perception tient à l'idée qu'on se fait généralement de la complicité, de la confidentialité entre deux amoureux, deux amis, deux associés en affaires. Selon notre conception contemporaine de l'individu, la confidentialité ne serait transférable que d'une individualité à l'autre. Mais à partir du moment où la lettre, plutôt que prise individuellement, c'est-à-dire comme un texte d'auteur seulement, est resituée dans la réalité de la polyphonie du réseau épistolaire, telle que constituée et ainsi significative pour ceux qui la créent, la vérité épistolaire, quant au contenu ou à l'interprétation des faits contenus dans les lettres, se présente historiquement tout autrement. Elle n'est plus dictée par les valeurs morales de l'historien ou de l'éditeur de lettres, mais 
simplement par ce qui se dit vrai ou faux dans un ensemble cohérent de lettres où les questions soulevées exigent des réponses. Ces lettres ont été écrites et lues et ont fonctionné, ont performé, diraiton aujourd'hui, ont provoqué d'autres lettres, d'autres pratiques, et c'est là que se trouve la véritable preuve de leur authenticité.

À l'intérieur d'un réseau effectif, les lettres racontent toutes le même événement, malgré les différences stylistiques qui cachent des découpages de la réalité et un ordre de présentation des choses particuliers; ce qui donne parfois l'impression qu'une lettre dit plus la vérité que l'autre. Les correspondants eux-mêmes n'ont pas la naïveté de croire en une seule vérité énonciative: "[...] c'était plaisir d'entendre ta version. Car chacun donne la sienne d'un événement, selon sa manière de voir, et ainsi c'est comme si on entendait une histoire différente chaque fois "59. Rien n'empêche l'histoire de mettre au jour l'événement biographique ou social en tenant compte des variations sur le même thème. L'accès prioritaire au plus grand nombre possible de réponses envoyées et reçues à l'intérieur du réseau devient alors plus important que le recours à n'importe quel autre document historique, si personnel soit-il, comme le journal ou les mémoires. La correspondance produit sa propre cohérence interne, son "système", pour reprendre l'expression de Bernard Bray évoquée au début, lequel donne sens et forme à l'événement. D'où l'importance de pouvoir se référer, chaque fois que cela est possible, à une correspondance générale.

Au lieu d'être à la recherche de fausses lettres, l'historien gagne à être l'affût de lettres qui ont la prétention naturelle d'être vraies même lorsqu'elles peuvent naturellement dire de gros mensonges. D'ailleurs, c'est ce qui fait l'objet de cette prétention qui est particulièrement significatif pour l'histoire des représentations et des échanges sociaux. Par exemple, la lettre où l'on raconte ses maladies en détail veut signifier la condition d'être malade plus que la maladie elle-même et c'est pourquoi elle ne prétend en aucun cas à être un traité médical. Du moins, est-elle ainsi lue. Il y aurait toute une histoire à faire des faussetés et irrégularités que l'histoire littéraire a cru découvrir - par exemple, l'affaire Casgrain dans l'histoire des livres de récompense ${ }^{60}$ - et

59 Gabrielle Roy, lettre à sa sœur Bernadette, 5 janvier 1966, op. cit., p. 113.

60 Réjean Robidoux, "Fortunes et infortunes de l'abbé Casgrain", dans Archives des lettres canadiennes, tome 1, Ottawa, Éditions de l'Université d'Ottawa, 1961, p. $79-99$. 
qui pourrait s'avérer fausse en regard de la perception que les écrivains d'une époque donnée ont eu de leurs propres pratiques et de celles de leurs contemporains. On s'étonnera du fait que la question soulevée par Réjean Robidoux en 1961 sur l'honnêteté de Casgrain n'ait pas encore été posée aux contemporains mêmes de Casgrain... L'édition et la lecture de la correspondance casgrainienne, reçue et envoyée, apportera sans aucun doute des nuances, en autant qu'on ne cherchât pas à vouloir à tout prix distinguer les vraies des fausses lettres en fonction de critères exogènes à la correspondance produite. Celles qui circulent ont bel et bien existé et leur édition n'exige aucune correction particulière d'interprétation. 